\title{
Disappearance of unilateral spatial neglect following a simple instruction
}

\author{
Sumio Ishiai, Keiko Seki, Yasumasa Koyama, Yorimichi Izumi
}

\begin{abstract}
Objectives-To clarify the reason why patients with left unilateral spatial neglect fail to copy the left side of a daisy like flower, not continuing to draw petals all around.

Methods-A flower was simplified and a figure was made that consisted of a large central circle and small circles surrounding it. Four patients with typical left unilateral spatial neglect performed copying and arrangement tasks to make this figure. In the arrangement task, they were instructed to arrange small circles all around the printed central circle.

Results-The patients' identification of the composition seemed flawless. In the copying task, they showed neglect, leaving a space on the left side. They seemed to adhere to their plan to place the same number of small circles as those of the model figure. By contrast, neglect disappeared in the arrangement task.

Conclusion-Patients with neglect can draw the figure satisfactorily if they use a spatial strategy to arrange small circles all around. This strategy seems to improve motivation for drawing and awareness for the left space. It is considered that in the copying of figures such as a daisy, failure to use a spatial strategy plays an important part in the appearance of left unilateral spatial neglect.
\end{abstract}

Department of Rehabilitation, Tokyo Metropolitan Institute for Neuroscience, 2-6 Musashidai, Fuchu City, Tokyo 183, Japan

S Ishiai

K Seki

Y Koyama

Department of Neurology,

Rehabilitation Centre

Kakeyu Hospital,

Nagano, Japan

Y Izumi

Correspondence to:

Dr Sumio Ishiai, Department

of Rehabilitation, Tokyo

Metropolitan Institute for

Neuroscience, 2-6

Musashidai, Fuchu City,

Tokyo 183, Japan.

Received 2 September 1996 and in final revised form 6 February 1997

Accepted 20 February 1997
(F Neurol Neurosurg Psychiatry 1997;63:23-27)

Keywords: unilateral spatial neglect; copy; spatial strategy

The copying of a flower such as a daisy is one of the simple and sensitive measures of unilateral spatial neglect. ${ }^{12}$ Patients with typical left unilateral spatial neglect copy the right sided petals and leave the left side unfinished. The horizontal extent occupied by the figure is much smaller than that of the stimuli for the other tests of neglect, such as the line cancellation $^{34}$ and line bisection ${ }^{5-7}$ tests. Object centred neglect for a rather small figure is often seen in copying, drawing, and perceptual tasks. ${ }^{8-10}$ However, there are only a few studies of the visuospatial process of copying a flower. ${ }^{11}{ }^{12}$ When asked to copy a flower, patients with neglect usually start to draw the right side, paying attention exclusively to the drawing site. Overattention to the right $\operatorname{side}^{13} 14$ and impaired disengagement ${ }^{15-17}$ from it may result in overlooking the left sided incompleteness.

In our previous study, ${ }^{12}$ all patients with neglect identified the stimulus figure as a flower that had petals all around the central circle. They never claimed that it lacked the left petals. Some of them, however, failed to notice the incompleteness when shown a flower without petals on the left side. Their mental image of a flower was therefore considered to be preserved and attentional mechanisms seem insufficient to explain neglect. As continuous drawing of petals to surround a central circle is also a simple spatial strategy to draw a flower, we expected that at least some patients with neglect could arrange petals all around when asked simply to do so, which would suggest that their defective copying resulted from failure to use this spatial strategy. To test this hypothesis, we developed copying and arrangement tasks, using a simplified figure that consisted of a large central circle and small circles surrounding it. We compared the performance of typical patients with neglect when copying the figure and performing the arrangement task that required them to arrange small circles all around the central circle.

\section{Patients and methods}

PATIENTS

Four right handed patients who showed severe left unilateral spatial neglect in the copying of a daisy with blades of grass on both sides were studied. ${ }^{611}$ The severity of neglect was measured using the line cancellation and line bisection tests which were rated as mild, moderate, or severe according to the scales of Levine et $\mathrm{l}^{18}$ and Ishiai et al. ${ }^{6}$ They showed mild neglect in the line cancellation test, ${ }^{34}$ as they omitted lines in the leftmost one third of the test sheet but crossed out all lines in the rightmost two thirds. In the line bisection test, ${ }^{67}$ one patient left less than one fifth of the line to the right of the subjective midpoint (severe neglect), two left more than one fifth but less than one third to the right (moderate neglect), and the remaining one left more than one third but less than a half to the right (mild neglect). All patients had left homonymous hemianopia on confrontation testing. Brain CT disclosed that three patients had a cerebrovascular lesion that mainly involved the right parietal lobe and the remaining one had an infarction of the anterior choroidal artery territory. ${ }^{19}{ }^{20}$ All had left hemiparesis and used the right hand to perform the screening tests for neglect and the experimental tasks. They gave informed consent to their 
Table 1 Clinical data and lesion sites of patients

\begin{tabular}{|c|c|c|c|c|c|c|c|}
\hline \multirow[b]{2}{*}{ Case } & \multirow{2}{*}{$\begin{array}{c}\text { Age } \\
(y)\end{array}$} & \multirow[b]{2}{*}{ Sex } & \multirow{2}{*}{$\begin{array}{l}\text { Duration } \\
\text { after onset } \\
\text { (months) }\end{array}$} & \multicolumn{3}{|l|}{$U S N$} & \multirow[b]{2}{*}{ Lesion } \\
\hline & & & & $\mathrm{CO}$ & $C A$ & $L B$ & \\
\hline 1 & 56 & M & 11.0 & +++ & + & + & FTPo (I) \\
\hline 2 & 74 & M & 4.0 & +++ & + & +++ & $\operatorname{Ach}(\mathrm{I})$ \\
\hline 3 & 80 & $\mathrm{~F}$ & 1.5 & +++ & + & ++ & Po $(\mathrm{H})$ \\
\hline 4 & 66 & $M$ & 2.0 & +++ & + & ++ & $\mathrm{fTP}(\mathrm{H})$ \\
\hline
\end{tabular}

participation in this study. Table 1 reports their clinical data and lesion sites.

METHODS

Copying task

We used as a model figure a simplified drawing of a daisy (figure A). A large central circle 35 $\mathrm{mm}$ in diameter surrounded by 10 small circles was centred on the stimulus card $(182 \times 57$ $\mathrm{mm})$. On the response sheet, only the large central circle was printed. The patients were presented with the stimulus card directly above the response sheet on the desk top so that each centre lay in the sagittal midplane of the trunk. The viewing distance was about $400 \mathrm{~mm}$. The examiner explained to the patients that the model figure had small circles all round the large central circle. Additionally, the figure was turned around to ensure the patients' understanding of its composition. The patients were then given the following instruction: "As you see, the central circle is already printed on the paper. Copy the model figure on this paper using the already printed central circle." The trial was ended when the patients said that they had finished. When they did nothing for 15 seconds, the examiner asked if they had completed the task. There was no time limit. The explanation about the model figure was repeated before each trial of the copying task.

\section{Arrangement task}

The same model figure and response sheet were presented to the patients in the same way as in the copying task. The examiner repeated the explanation about the model figure, turning around it, and then gave the following instruction: "As you see, the central circle is already printed on the paper. Arrange small circles all around the printed circle as you find in the model figure." The trial was ended when the patients said that they had finished. When no response occurred for 15 seconds, they were asked if they had completed the task. There was no time limit.

\section{Discrimination task}

The ability of the patients to discriminate between the presence and absence of the right or left sided small circles of the figure was also examined. Three stimulus figures were used. The perfect figure was the same as the model used for the copying and arrangement tasks. The "left missing" figure was made by removing the leftmost three circles from the model figure, and the "right missing" figure was made
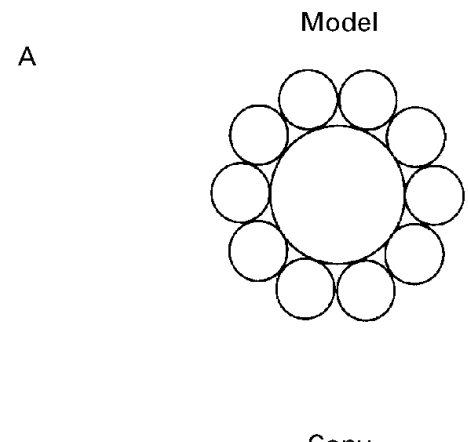

B
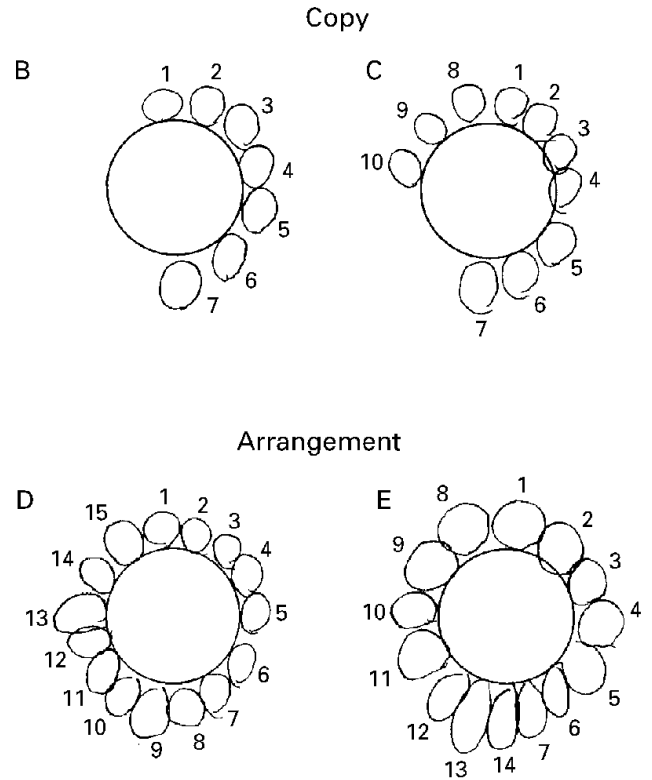

Examples of drawings for copying and arrangement tasks (patient 1). The numbers around each drawing show the order in which the small circles were placed.

by removing the rightmost three circles. Each figure was centred on a card $(182 \times 257 \mathrm{~mm})$. Before starting the experimental trials, the examiner presented each of the three figures and, for the left missing or right missing figures, pointed at the three missing circles. The examiner then presented one figure at a time and asked the patient if the figure was perfect, or missing the right circles, or the left circles. The centre of each figure was positioned in the sagittal midplane of the trunk. No time limit was imposed and there was no restriction of head or eye movements. The viewing distance was the same as that for the copying and arrangement tasks. The patients performed 20 trials for each figure in randomised order.

All patients initiated the experimental session with the first block of drawing. They performed four trials for each of the copying and arrangement tasks. The order of the two tasks was counterbalanced with an ABBA design. They were then given the discrimination task. Three patients underwent the second block of drawing, thereby completing eight trials in total for each of the copying and arrangement tasks. The remaining patient (patient 4) refused further drawing because of fatigue. At the end of the session, the examiner showed the patients their own drawings and asked if they were per- 
Table 2 Performances in the copying and arrangement tasks

\begin{tabular}{|c|c|c|c|c|c|c|c|c|}
\hline \multirow[b]{3}{*}{ Case } & \multicolumn{4}{|l|}{ Copy } & \multicolumn{4}{|l|}{ Arrangement } \\
\hline & \multirow{2}{*}{$\begin{array}{l}\text { Occurrence of } \\
\text { neglect }\end{array}$} & \multicolumn{2}{|c|}{ Number of circles } & \multirow[b]{2}{*}{ Latency (s) } & \multirow{2}{*}{$\begin{array}{l}\text { Occurrence of } \\
\text { neglect }\end{array}$} & \multicolumn{2}{|c|}{ Number of circles } & \multirow[b]{2}{*}{ Latency (s) } \\
\hline & & Left & Right & & & Left & Right & \\
\hline 1 & $8 / 8$ & $3.3(1.2)$ & $6.5(0.8)$ & $18.0(7.1)$ & $0 / 8$ & $7.5(1.5)$ & $7.3(0.9)$ & $3.6(1.3)$ \\
\hline 2 & $8 / 8$ & $0.9(0.2)$ & $4.5(0.5)$ & $7.9(2.5)$ & $0 / 8$ & $5.1(0.9)$ & $5.3(0.7)$ & $2.8(1.5)$ \\
\hline 3 & $6 / 8$ & $3.6(1.1)$ & $5.5(0.7)$ & $9.8(6.6)$ & $0 / 8$ & $6.3(0.6)$ & $5.7(0.6)$ & $0.9(0.4)$ \\
\hline 4 & $4 / 4$ & $3.3(0.5)$ & $7.0(2.2)$ & $10.1(2.4)$ & $0 / 4$ & $6.3(0.5)$ & $7.3(1.0)$ & $2.0(1.1)$ \\
\hline
\end{tabular}

For number of circles and latency, values are means (SD).

fect or had any mistakes. All performances were recorded with a videotape recorder.

\section{Results}

NEGLECT IN COPYING AND ARRANGEMENT TASKS Table 2 presents the results of the two tasks for each patient. The right side of each drawing was satisfactory in both copying and arrangement: Small circles were compactly placed outside the central circle. On the left side of the copies, a gap was often left between the small circles (figure B and C). Each gap had space enough for two or more circles of the average size in each copy. This was regarded as evidence of left unilateral spatial neglect. By contrast, no apparent gap or neglect was found in the drawings made during the arrangement task (figure D and E). Patients 1, 2, and 4 exhibited neglect in all trials of the copying task whereas they performed almost satisfactorily in all trials of the arrangement task. Slight neglect was suspected in only one arrangement trial in patient 1 , as a small gap enough to place a small circle was visible on the left side. In patient 3, neglect was evident in six of the eight copying trials but in none of the arrangement trials. This difference in the occurrence of neglect was significant (Fisher's exact test, $\mathrm{P}<0.01$ ).

The number of small circles on the left side and that on the right side were separately counted for each drawing. When a circle was positioned just above or beneath the central circle, one half count was added to either the right or left count. Table 2 shows the mean number on each side for the copying and arrangement tasks for each patient. In the copying task, the mean number of circles was greater on the right side than on the left for all patients. This difference was significant for patients 1, 2, and 3 (Wilcoxon matched pairs signed rank test, $\mathrm{P}<0.025)$. For patient 4 , it fell a little short of the significance level because of small sample size $(P=0.066)$. In the arrangement task, the mean number on the left side was not significantly different from that on the right for patients 1 and 2 . For patient 3 , the mean number of circles on the left side was greater than that on the right $(\mathrm{P}<0.05)$. Patient 4 drew six or more circles compactly on either side, although the mean number tended to be greater on the right $(\mathrm{P}=0.10)$.

ATTITUDE BEFORE DRAWING

The latency to start drawing after the examiner's instruction was measured for each trial, reproducing the videotape recordings. The mean latency was significantly longer in the copying task than in the arrangement task for each patient (Mann-Whitney $U$ test, $\mathrm{P}<0.025$ ).

When asked to copy the model figure, all patients seemed to count the number of small circles by moving the pencil, although the examiner did not require them to do so. Patient 2 said "five circles" and drew a total of five or six circles in five trials. Patient 3 placed eight or 10 circles according to her count in three trials. In two trials, however, she drew eight or 11 circles saying "seven" as she showed neglect for her own drawings. Patient 4 counted 10 and drew 10 circles in one trial. In the arrangement task, all patients showed no gesture of counting and said nothing before they started to draw circles.

\section{ORDER OF DRAWING CIRCLES}

Copying started with the top circle and went on to the right side in three patients. Patient 1 placed the circles clockwise in four trials (figure B), clockwise and counterclockwise in three trials (figure C), and in unsystematic order in one trial. Patient 2 placed the circles clockwise in five trials and in unsystematic order in three trials. Patient 4 always proceeded clockwise. Only patient 3 started with the left upper circle and placed the others clockwise. In the arrangement task, three trials of patient 1 , two trials of patient 2 , and all four trials of patient 4 were started clockwise from the top placing circles all around (figure D). Patient 3 completed all eight trials in a similar way except that she started with the left upper circle. In two trials of patient 1 and six trials of patient 2, circles were arranged clockwise on the right side and then counterclockwise on the left side (figure E). Three trials of patient 1 were initiated by placing four circles at the locations of $12,3,6$, and 9 o'clock before drawing the other circles.

APPRECIATION OF ERRORS AFTER A DELAY

Patients 2 and 3 failed to notice the gap made on the left side in all but one of their copies. Patient 4 overlooked the gap in all copies. Patients often referred to the inaccuracy on the right side, such as distortion, inappropriate size, and wrong number of small circles. Patient 1 always found the error and pointed to the gap.

DISCRIMINATION TASK

All patients correctly responded to the right missing figure. Patients 1 and 4 completely dis- 
criminated between the left missing and perfect figures. The performance of patient 3 was also satisfactory except in one trial where he regarded the perfect figure as the left missing one. Patient 2 correctly responded to the perfect figure but failed to notice the absence of the left circles in six of the 20 trials.

\section{Discussion}

The four patients showed left unilateral spatial neglect when asked to copy a figure that consisted of a large central circle and small circles surrounding it. Their copies lacked small circles on the left side. By contrast, as we expected, they could arrange small circles all around the central circle when instructed simply to do so, and they exhibited no apparent neglect. The patients were able to discriminate between the presence and absence of the left sided circles of the figures prepared by the examiner, as reported by Ishiai et al. ${ }^{12}$ It is therefore unlikely that neglect found in the copying resulted from misperception of the left side of the model figure. The copying and arrangement tasks required the same response and we think that the contrasting performances can be explained as a result of the different instructions.

The patients with neglect used a spatial strategy to place small circles all around the central circle following the instruction for the arrangement task, showing two main patterns of arrangement. In the first pattern, found in about $60 \%$ of the trials, patients simply placed small circles clockwise. A similar effect was also described by Ishiai et $a l^{4}$ who reported that numbering instead of crossing out improved neglect in the line cancellation test. They attributed this improvement to the motivational effect of numbering. The instruction to place circles all around may also have motivated the patients to continue drawing until they went around and returned to find the initial circle. The second pattern was seen in some trials of two patients. They placed circles clockwise on the right side and then counterclockwise on the left side. Awareness for the left side of the image ${ }^{2122}$ seemed to be spared when following the instruction for the arrangement task.

We found an unusual pattern of arrangement in three trials of one patient (patient 1) who placed four circles at the locations that corresponded to $12,3,6$, and 9 o'clock and then drew the remaining ones. His verbal IQ was 91 on the Wechsler adult intelligence scale-revised. ${ }^{23}$ Preserved verbal intelligence may have facilitated the use of planning in a way similar to that reported in the clock drawing of some patients with neglect. ${ }^{24}$

The apparent neglect found in the left side of the copies suggests that patients did not use a spatial strategy. For either task, the examiner did not require them to place the same number of circles as those of the model figure. Following the instruction to copy, however, the patients spent time enough to count the number of small circles in the model figure. They seemed to adhere to their plan to place small circles according to the count. The orders of copying were similar to those of the arrangement trials in that the right space was filled first. The adherence to the count thus resulted in leaving a gap on the left side. When shown the copies after a delay, the patients pointed out the distortion or inappropriate size of the small circles. They might have tried to reproduce the small circles exactly in the copying process. The instruction to copy was probably more demanding than that used for the arrangement task. Right hemispheric damage may reduce the capacity for activation that is necessary for parallel execution of two tasks. ${ }^{25}$ We consider that patients with neglect find it difficult to use the spatial strategy when they are trying to reproduce some partial feature of the model figure.

Our patients never noticed the left sided incompleteness of their copy when they finished the trial. However, they noticed the left sided incompleteness of the figures prepared by the examiner. These findings replicated the results of the study of Ishiai et al, ${ }^{12}$ which suggested that leftward shift of attention may take place during free observation of the figure, whereas the action of copying may worsen this shift. They considered that the close integration of attentional and premotor mechanisms ${ }^{26}$ may be crucial for the appearance of neglect in copying a single object. The results of our study suggest that failure to use a spatial strategy may also play a part in the defective shift of attention to the left side.

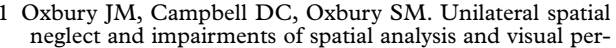
neglect and impairments of spatial
ception. Brain 1974;97:551-64.

2 Heilman KM, Watson RT, Valenstein E. Neglect and related disorders. In: Heilman KM, Valenstein E, eds. Clinical neuropsychology, 2nd ed. New York: Oxford University Press, 1985:243-93.

3 Albert ML. A simple test of visual neglect. Neurology 1973; 23:658-64.

4 Ishiai S, Sugishita $M$, Odajima N, Yaginuma $M$, Gono $S$, Kamaya T. Improvement of unilateral spatial neglect with numbering. Neurology 1990;40:1395-8.

5 Schenkenberg T, Bradford DC, Ajax ET. Line bisection and unilateral visual neglect in patients with neurologic impairment. Neurology 1980;30:509-17.

6 Ishiai S, Furukawa T, Tsukagoshi H. Visuospatial processes of line bisection and the mechanisms underlying unilateral of line bisection and the mechanisms underly

7 Ishiai S, Sugishita M, Watabiki S, Nakayama T, Kotera M, Ishiai S, Sugishita $M$, Watabiki S, Nakayama T, Kotera $M$,
Gono S. Improvement of left unilateral spatial neglect in a line extension task. Neurology 1994;44:294-8.

8 Driver J, Halligan PW. Can visual neglect operate in objectcentred co-ordinates? An affirmative single-case study. Cognitive Neuropsychology 1991;8:475-96.

9 Marshall JC, Halligan PW. Visuo-spatial neglect: a new copying test to assess perceptual parsing. If Neurol 1993;240:37-40.

10 Halligan PW, Marshall JC. When two is one: a case study of spatial parsing in visual neglect. Perception 1993;22:309-12.

11 Seki K, Ishiai S, Koyama Y, Fujimoto Y. Appearance and disappearance of unilateral spatial neglect for an object: influence of attention-attracting peripheral stimuli. Neuropsychologia 1996;34:819-26.

12 Ishiai S, Seki K, Koyama Y, Yokota T. Mechanisms of unilateral spatial neglect in copying a single object. Neuropsychologia 1996;34:965-71.

13 Mark VW, Kooistra CA, Heilman KM. Hemispatial neglect affected by non-neglected stimuli. Neurology 1988;38: 1207-11.

14 Kinsbourne M. Mechanisms of unilateral neglect. In: Jeannerod $\mathrm{M}$, ed. Neurophysiological and neuropsychological aspects of spatial neglect. Amsterdam: Elsevier, 1987;69-86. parietal injury on covert orienting of attention. $\mathcal{F}$ Neurosci 1984;4:1863-74.

16 Làdavas E, Umiltà C, Ziani P, Brogi A, Minarini,M. The role of right side objects in left side neglect: a dissociation between perceptual and directional motor neglect. Neuropsychologia 1993;31:761-73.

17 Di Pellegrino G. Clock-drawing in a case of left visuo-spatial neglect: a deficit of disengagement? Neuropsychologia 1995; 33:353-8 
18 Levine DN, Warach JD, Benowitz L, Calvanio R. Left spatial neglect: effects of lesion size and premorbid brain atrophy on severity and recovery follow

19 Ferro JM, Kertesz A. Posterior internal capsule infarction associated with neglect. Arch Neurol 1984;41:422-4.

20 Bogousslavsky J, Miklossy J, Regli F, Deruaz J-P, Assal G Delaloye B. Subcortical neglect: neuropsychological, SPECT, and neuropathological correlations with anterior choroidal artery territory infarction. Ann Neurol 1988;23 448-52. 21 Anderson B. Spared awareness for the left side of internal
visual images in patients with left-sided extrapersonal neglect. Neurology 1993;43:213-6.
22 Bartolomeo P, D'Erme P, Gainotti G. The relationship between visuospatial and representational neglect. Neurolbetween visuospatial

ogy 1994:44:1710-4.
23 Weschler D the Weschler adult intelligence scale-revised: Japanese edition. Tokyo: Nihon Bunka Kagakushaa, 1990. 24 Ishiai S, Sugishita, M, Ichikawa,T, Gomno S, Watabiki S. Clock-drawing test and unilateral spatial neglect. Neurology 1993;43:106-10.

25 Coslett HB, Bowere D, Heilman KM. Reduction in cerebral activation after right hemisphere stroke. Neurology 1987;37: 957-62.

26 Rizzolatti G, Gallese V. Mechanisms and theories of spatial psychology. Vol 1. Amsterdam: Elsevier, 1988:223-46. 